\title{
Un ringraziamento ai Revisori dei manoscritti inviati a La Rivista Italiana della Medicina di Laboratorio-Italian Journal of Laboratory Medicine nel biennio 2017-2018
}

\author{
Piero Cappelletti $^{1} \cdot$ Nicola Bizzaro $^{2} \cdot$ Romolo M. Dorizzi $^{3}$
}

Pubblicato online: 21 novembre 2018

(c) Società Italiana di Patologia Clinica e Medicina di Laboratorio 2018

La revisione tra pari dei manoscritti inviati ai giornali scientifici non è fondamentale solo per il loro successo ma anche per la loro stessa sopravvivenza. Anche nel biennio 2017-2018 decine di colleghi hanno messo a disposizione il loro tempo, la loro cultura e la loro esperienza professionale per la revisione dei manoscritti che sono stati inviati per la pubblicazione.

Desideriamo ringraziare, a nome degli autori, tutti i colleghi sottoelencati per l'aiuto e il fondamentale contributo alla qualità del giornale.

Piero Cappelletti, Direttore Responsabile

Nicola Bizzaro, Direttore Scientifico

Romolo M. Dorizzi, Direttore Scientifico

Aita Ada, Italia

Amendola Alessandra, Italia

Bagnasco Marcello, Italia

Bondanini Francesco, Italia

Boscutti Giuliano, Italia

Caleffi Alberta, Italia

Camerotto Alessandro, Italia

Caponi Laura, Italia

Castaman Giancarlo, Italia

Cuda Giovanni, Italia

Cuomo Laura, Italia

Dall'Olio Giuliano, Italia

Daves Massimo, Italia

Del Mistro Annarosa, Italia

Deleonardi Gaia, Italia

Fabris Martina, Italia

Facchinetti Roberto, Italia

Fontanini Elisabetta, Italia
Gessoni Gianluca, Italia

Guerranti Roberto, Italia

Gulletta Elio, Italia

Longini Mariangela, Italia

Luzza Francesco, Italia

Malanga Donatella, Italia

Manoni Fabio, Italia

Mezzapelle Giacomo, Italia

Miconi Valentino, Italia

Modolo Maria Luisa, Italia

Montalcini Tiziana, Italia

Morandini Margherita, Italia

Morelli Benedetto, Italia

Moretti Marco, Italia

Padoan Adrea, Italia

Papa Fabrizio, Italia

Pasini Luciano, Italia

Pentimalli Henny, Italia
Pierangeli Alessandra, Italia

Porzio Ottavia, Italia

Pradella Marco, Italia

Rossi Luca, Italia

Ruscio Maurizio, Italia

Santoro Cristina, Italia

Sargentini Valeria, Italia

Steffan Agostino, Italia

Stellini Roberto, Italia

Stenner Elisabetta, Italia

Terzuoli Lucia, Italia

Tozzoli Renato, Italia

Vannoni Daniela, Italia

Vero Anna, Italia

Villalta Danilo, Italia

Vitillo Marina, Italia

Zandonà Lorenzo, Italia

Zuccherini Mario, Italia

\footnotetext{
P. Cappelletti

pie.cappelletti@gmail.com

1 SIPMeL, Via Ponchini 17/7, 31033 Castelfranco Veneto, TV, Italia

2 Laboratorio di Patologia Clinica, Ospedale Sant'Antonio, Tolmezzo, UD, Italia

3 U.O. Patologia Clinica-Laboratorio Unico, AUSL della Romagna, Pievesestina di Cesena, FC, Italia
} 